\section{A novel, noncanonical mechanism of cytoplasmic polyadenylation operates in Drosophila embryogenesis}

\author{
Olga Coll, ${ }^{1}$ Ana Villalba, ${ }^{1}$ Giovanni Bussotti, ${ }^{2}$ \\ Cedric Notredame, ${ }^{2}$ and Fátima Gebauer ${ }^{1,3}$ \\ ${ }^{1}$ Gene Regulation Programme, Centre de Regulació Genòmica \\ (CRG-UPF), 08003 Barcelona, Spain; ${ }^{2}$ Bioinformatics \\ Programme, Centre de Regulació Genòmica (CRG-UPF), 08003 \\ Barcelona, Spain
}

Cytoplasmic polyadenylation is a widespread mechanism to regulate mRNA translation that requires two sequences in the $3^{\prime}$ untranslated region (UTR) of vertebrate substrates: the polyadenylation hexanucleotide, and the cytoplasmic polyadenylation element (CPE). Using a cell-free Drosophila system, we show that these signals are not relevant for Toll polyadenylation but, instead, a "polyadenylation region" $(\mathrm{PR})$ is necessary. Competition experiments indicate that PR-mediated polyadenylation is required for viability and is mechanistically distinct from the $\mathrm{CPE} /$ hexanucleotide-mediated process. These data indicate that Toll mRNA is polyadenylated by a noncanonical mechanism, and suggest that a novel machinery functions for cytoplasmic polyadenylation during Drosophila embryogenesis.

Supplemental material is available at http://www.genesdev.org.

Received July 20, 2009; revised version accepted November 23, 2009 .

Oocyte maturation and early development in many organisms occurs in the absence of transcription. Developmental progression at these times depends largely on differential translation of maternal mRNAs, and cytoplasmic polyadenylation is a major component of this control. In general, mRNAs with a short poly(A) tail remain translationally silent, while elongation of the poly(A) tail in the cytoplasm results in translational activation. Most of the accumulated knowledge on cytoplasmic polyadenylation derives from studies in oocytes of Xenopus (for review, see Belloc et al. 2008; Radford et al. 2008). Two cis-acting sequences in the $3^{\prime}$ untranslated region (UTR) of substrate mRNAs are essential for this process: the conserved polyadenylation hexanucleotide -also required for nuclear polyadenylation-with the structure A(A/U)UAAA, and the U-rich cytoplasmic polyadenylation element (CPE), which generally consists of $\mathrm{U}_{4-5} \mathrm{~A}_{1-3} \mathrm{U}$. The hexanucleotide is recognized by the multisubunit complex CPSF (cleavage and polyadenylation specificity factor), and the CPE is recognized by

[Keywords: CPE; hexanucleotide; polyadenylation; Toll]

${ }^{3}$ Corresponding author.

E-MAIL fatima.gebauer@crg.es; FAX 34-93-3969983.

Article is online at http://www.genesdev.org/cgi/doi/10.1101/gad.568610.
$\mathrm{CPEB}$, a protein with a dual function that acts as a switch between translational repression and activation. In immature oocytes, CPEB represses translation by recruiting a set of factors that functionally block the two ends of the mRNA. On the one hand, CPEB recruits Maskin (or 4E-T in growing oocytes), which in turn binds to eIF4E and prevents its recognition by eIF4G during translation initiation (Stebbins-Boaz et al. 1999; Minshall et al. 2007). On the other hand, CPEB recruits the deadenylase PARN, which keeps the poly(A) tail short (Kim and Richter 2006). Upon meiotic maturation, CPEB phosphorylation results in eviction of PARN and enhanced recruitment of CPSF. Together, CPEB and CPSF recruit the cytoplasmic poly(A) polymerase GLD-2, leading to elongation of the poly (A) tail and translational activation (Barnard et al. 2004). The distance between the CPE and the hexanucleotide dictates the timing and extent of polyadenylation (Piqué et al. 2008). Additional elements reported to function early during oocyte maturation are the U-rich polyadenylation response elements (PREs), which bind the protein Musashi /Charlesworth et al. 2002, 2006).

CPEB is a conserved family of four members in vertebrates that, in addition to oocyte maturation, contribute to the regulation of local protein synthesis at synapses that underlies long-term changes in synaptic plasticity (for review, see Richter 2007). In Drosophila, the CPEB1 homolog Orb plays a role in mRNA localization and regulates the polyadenylation of oskar and cyclin $B$ mRNAs during oogenesis (Chang et al. 1999; Castagnetti and Ephrussi 2003; Benoit et al. 2005). Orb2, the homolog of CPEB2-4, is required for long-term memory, but its role in cytoplasmic polyadenylation has not been demonstrated (Keleman et al. 2007). Other conserved factors that contribute to cytoplasmic polyadenylation during Drosophila oogenesis are the canonical poly(A) polymerase Hiiragi, and the GLD-2 homolog Wispy (Wisp) (Juge et al. 2002; Benoit et al. 2008; Cui et al. 2008).

Cytoplasmic polyadenylation also occurs during embryogenesis, but the sequences and factors responsible for polyadenylation at these times remain poorly understood. In Drosophila, translation of the transcripts encoding Bicoid, Toll, and Torso is activated by cytoplasmic polyadenylation in early embryogenesis, and this activation is required for appropriate axis formation (Sallés et al. 1994; Schisa and Strickland 1998). How this polyadenylation occurs is intriguing, as Orb is barely detectable in early embryos (Vardy and Orr-Weaver 2007). Furthermore, no cis-acting elements responsible for cytoplasmic polyadenylation have been described yet in this organism. Therefore, an important question is whether similar signals, factors, and mechanisms operate for cytoplasmic polyadenylation in different biological settings. To address this question, we used an in vitro cytoplasmic polyadenylation system derived from Drosophila early embryos. Using Xenopus cyclin B1 (CycB1) mRNA as a substrate, we found that the canonical cytoplasmic polyadenylation signals-the CPE and the hexanucleotide-function in Drosophila. Surprisingly, however, these sequences are not necessary for Toll polyadenylation. Rather, a region of the $3^{\prime}$ UTR that we term the "polyadenylation region" (PR) is required. Consistently, competition experiments indicate 
that PR-mediated polyadenylation is mechanistically distinct from the CPE/hexanucleotide-mediated process, implying that a novel machinery for cytoplasmic polyadenylation operates during Drosophila embryogenesis.

\section{Results and Discussion}

\section{A Drosophila cell-free system for cytoplasmic polyadenylation}

Cytoplasmic polyadenylation has been observed in oocytes and early embryos of Drosophila. Maximal polyadenylation of Toll and bicoid mRNAs occurs at $\sim 90 \mathrm{~min}$ of development (Sallés et al. 1994). To study cytoplasmic polyadenylation, we first tested whether extracts from 90-min embryos could recapitulate this process. Similar extracts obtained from nonstaged embryos have been shown previously to support translation /Gebauer et al. 1999). Because translation is the consequence of cytoplasmic polyadenylation for Toll and bicoid, we tested whether polyadenylation of these substrates in embryonic extracts could occur under translation conditions. We incubated nonadenylated full-length bicoid mRNA and the 3' UTR of Toll in staged 90-min embryo extracts. In addition, we used the mRNA encoding the ribosomal protein Sop as a negative control, as this transcript contains a canonical hexanucleotide and has been shown to undergo nuclear but not cytoplasmic polyadenylation (e.g., see Benoit et al. 2008). After incubation, we measured the length of the poly(A) tail by the PCR-based poly(A) test (PAT) assay. We found that both bicoid and Toll RNAs gained a poly(A) tail of $\sim 150$ nucleotides (nt) while sop mRNA remained nonadenylated, indicating that this system recapitulates the cytoplasmic polyadenylation process (Fig. 1A). Toll mRNA was selected for further studies because it was consistently polyadenylated more efficiently than bicoid mRNA in the cell-free system.

To evaluate whether cytoplasmic polyadenylation resulted in increased translation, we first tested the correlation between both processes in a time-course experiment. We fused the 3' UTR of Toll to the firefly luciferase ORF to yield the Luc-toll transcript. Translation of this transcript closely paralleled polyadenylation of Toll 3' UTR (Fig. 1B, cf. the left panel and the Luc-toll curve in the right panel). In addition, treatment of the mRNA with the chain elongation inhibitor cordycepin (3'-deoxyadenosine) dramatically reduced the efficiency of translation, decreasing it to the levels of nonadenylated luciferase mRNA(Fig. 1B, right panel). These data show that both cytoplasmic polyadenylation and polyadenylationdependent translation can be recapitulated in 90-min Drosophila embryo extracts.

\section{The canonical cytoplasmic polyadenylation signals function in Drosophila}

The cis-acting elements for cytoplasmic polyadenylation in Drosophila are unknown. To test whether the CPE and the hexanucleotide were recognized as polyadenylation elements in this organism, we analyzed the polyadenylation of the best-characterized vertebrate substrate, CycB1. The 3' UTR of this transcript contains three CPEs, one of them overlapping with the hexanucleotide (Fig. 1C), and has been shown to undergo strong poly-

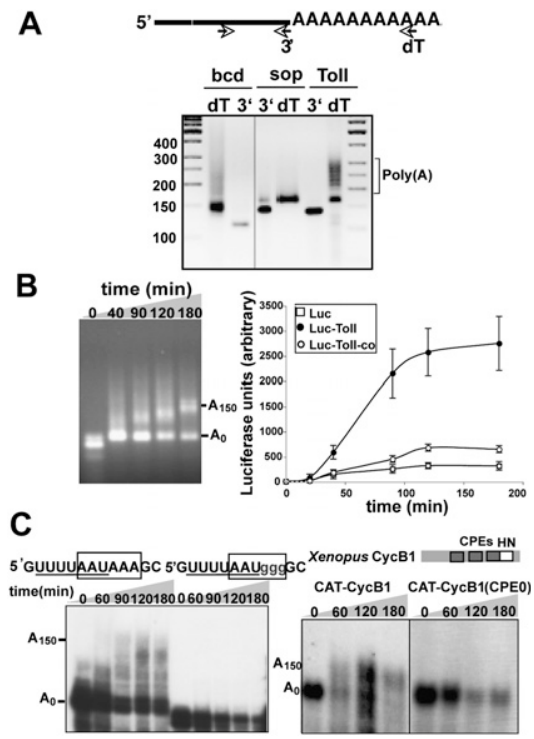

Figure 1. The canonical cytoplasmic polyadenylation signals function in Drosophila. (A) Cytoplasmic polyadenylation in Drosophila embryo extracts. Nonadenylated full-length sop and bcd mRNAs, as well as the $3^{\prime}$ UTR of Toll, were incubated in 90-min embryo extracts, and the poly(A) tail was measured using the PAT assay. A schematic representation of the oligonucleotides used in this assay is shown in the top panel. For each transcript, a specific $5^{\prime}$ oligonucleotide was combined with either a specific $3^{\prime}$ primer to reveal the size of the nonadenylated RNA ( $3^{\prime}$ lanes), or with an oligo(dT) anchor to visualize the poly(A) tail (dT lanes). Molecular weight markers are also shown. $(B)$ Cytoplasmic polyadenylation promotes translation of Toll mRNA. (Left panel) Polyadenylation time course of the Toll 3' UTR, as measured by PAT assay. (Right panel) A firefly luciferase reporter containing the $3^{\prime}$ UTR of Toll (Luc-toll) was incubated in embryo extracts for different times, and the efficiency of translation was determined by measuring the luciferase activity. As controls, the translation efficiencies of nonadenylated Luciferase and cordycepin-treated Luc-toll mRNAs were determined. Curves represent the average of five independent experiments. (C) The CPE and the hexanucleotide are functional CPEs in Drosophila. (Left panel) Polyadenylation of radioactively labeled wild-type and hexanucleotide-mutated $C y c B 13^{\prime}$ UTRs. The nature of the mutation is indicated in gray lowercase letters. RNAs were separated in a $6 \%$ denaturing acrylamide gel and visualized using the PhosphorImager. (Right panel) Polyadenylation of CAT reporter mRNAs containing either a wild-type $C y c B 133^{\prime}$ UTR or a derivative with U-to-G transversions in all three CPEs. RNAs were visualized by Northern blot using a probe against the CAT ORF. A schematic representation of a Xenopus CycB1 3' UTR is shown, with the three CPEs and the polyadenylation hexanucleotide (HN) depicted as gray and white boxes, respectively.

adenylation at a late time during Xenopus oocyte maturation and in early embryos (Groisman et al. 2002; Piqué et al. 2008). The 3' UTR of CycB1 was polyadenylated in the Drosophila cell-free system and was sufficient to confer polyadenylation when fused to a CAT reporter (Fig. 1C). Importantly, mutation of the hexanucleotide (Fig. 1C, left panel) or the CPEs (Fig. 1C, right panel, CPEO) completely abrogated polyadenylation, indicating that the vertebrate cytoplasmic polyadenylation signals function in Drosophila. These results suggest that a canonical cytoplasmic polyadenylation machinery exists in Drosophila embryos. Intriguingly, however, poly(A) tail length control must occur in the absence of Orb, which is undetectable in early embryos, and PARN, which is not conserved in Drosophila. 
Toll $m R N A$ is polyadenylated in a CPE-independent and hexanucleotide-independent fashion

Toll mRNA contains a canonical hexanucleotide, followed by a putative CPE (Fig. 2A). To determine whether these sequences were responsible for polyadenylation, we analyzed the behavior of Toll mutant derivatives. To visualize the polyadenylated products, we used Northern blots, which allow a more accurate measurement of the efficiency of polyadenylation as compared with PAT assays. Surprisingly, mutation or deletion of the CPE and/or the hexanucleotide did not affect polyadenylation of Toll (Fig. 2A). Treatment with oligo(dT) and RNase H confirmed that the size increase of Toll upon incubation was due to polyadenylation (Supplemental Fig. 1). That Toll polyadenylation was unaffected by deletion of the CPE and the hexanucleotide was unexpected, as both elements function as polyadenylation signals in Drosophila (Fig. 1C), and a single point mutation in the hexanucleotide (AAUAAA to AAGAAA) is sufficient to hinder cytoplasmic polyadenylation in vertebrates (e.g., see McGrew and Richter 1990). Thus, it appeared that Toll polyadenylation was independent of the CPE and the hexanucleotide. However, the canonical polyadenylation machinery could, in principle, bind to functional variations of these elements that could pass unrecognized by sequence inspection. To exclude this possibility, we performed competition assays. СусB1 effectively competed the polyadenylation of radiolabeled $C A T-C y c B 1$ (Fig. 2B, lanes 10-15). Polyadenylation of Toll was readily competed by an excess of cold Toll 3' UTR, but, re-

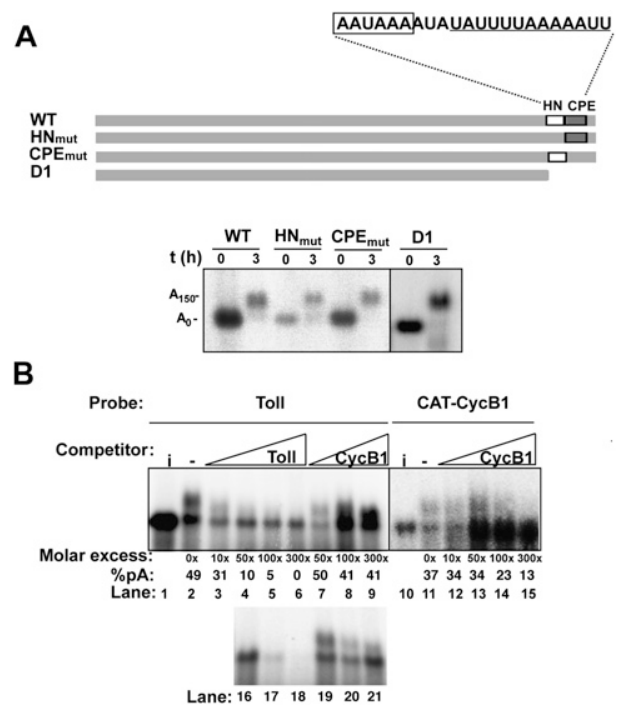

Figure 2. Polyadenylation of Toll mRNA is independent of the CPE and the hexanucleotide. (A, top panel) Schematic representation of a Toll 3' UTR (1256 nt) and mutant derivatives. The location and sequence of the putative CPE and the hexanucleotide is detailed. (Bottom panel) Polyadenylation of these mRNAs was measured by Northern blot. $(B)$ Polyadenylation competition assays. Polyadenylation of a ${ }^{32}$ P-labeled Toll 3' UTR or CAT-CycB1 after addition of excess Toll or $\mathrm{CycB} 13^{\prime}$ UTRs. RNAs were separated in a $1 \%$ denaturing agarose gel. Input (i) RNA probes are also shown. The percentage of polyadenylated transcript with respect to total transcript within each lane is indicated (\%pA). Lanes 16-21 in the bottom panel show samples of the same reactions as lanes 4-9 taken 15 min later. markably, not by an excess of $C y c B 1$ (Fig. 2B, lanes 1-9, and see also lanes 16-21, showing the same reactions as lanes 4-9 taken 15 min later). These data argue that polyadenylation of Toll is independent of the CPE and the hexanucleotide. Addition of excess Toll competitor also destabilized the Toll substrate, while addition of $C y c B 1$ did not (Fig. 2B, lanes 16-21). In addition, nonadenylated $C A T-C y c B 1$ was often stabilized in the presence of $C y c B 1$ competitor (Fig. 2B, lanes 13-15), suggesting that the Drosophila extracts can be used to monitor both stability and adenylation changes, but that these two processes are not necessarily linked.

\section{A proximal region in Toll 3' UTR directs noncanonical cytoplasmic polyadenylation}

To identify the elements of Toll that were responsible for cytoplasmic polyadenylation, we performed mutational analysis. The distal $40 \%$ of Toll 3' UTR could be deleted without significant consequences for cytoplasmic polyadenylation (Fig. 3A, fragment D3). Further deletions progressively reduced the efficiency of polyadenylation (fragments D4 and D5). A region of $183 \mathrm{nt}$ within the first half of the $3^{\prime}$ UTR was sufficient to provide detectable levels of polyadenylation (fragment D6), while other regions of the 3' UTR were not (fragments D5 and D7). We refer to the D6 fragment as the PR. Importantly, deletion of the PR from an otherwise wild-type 3 ' UTR severely blocked polyadenylation and translation of Toll, indicating that the PR is essential for expression of this mRNA (Fig. 3B).

Although the PR is necessary for polyadenylation, other sequences within the Toll 3' UTR seem to influence both the efficiency of polyadenylation and the length of the poly(A) tail. Deletions downstream from the PR reduce the polyadenylation efficiency, while deletions upstream of the PR reduce the length of the poly(A) tail (Fig. 3A, cf. fragments D3, D4, and D6). This illustrates the complexity and fine regulation of the process, which is likely to be mediated by a complex interplay of multiple activities. Auxiliary elements located both upstream of and downstream from the polyadenylation signal have also been described for nuclear polyadenylation (Chen and Wilusz 1998; Zarudnaya et al. 2003), including sequences and factors that mediate hexanucleotideindependent polyadenylation (Venkataraman et al. 2005).

Elements other than the canonical CPE have been shown previously to stimulate cytoplasmic polyadenylation in other organisms. In Xenopus oocytes, the U-rich PRE and TCS (translational control sequence) stimulate the polyadenylation of a number of mRNAs early after progesterone induction (Charlesworth et al. 2002, 2004; Wang et al. 2008). Similarly, poly(U) and poly(C) sequences promote polyadenylation during Xenopus embryogenesis (Simon et al. 1992; Paillard et al. 2000), and undefined elements other than the CPE and the hexanucleotide direct polyadenylation of lamin B1 mRNA in Xenopus embryos (Ralle et al. 1999). However, no direct evidence exists that these elements function independently of the canonical polyadenylation machinery. To confirm that the $\mathrm{PR}$ is responsible for noncanonical polyadenylation, we performed competition assays. Polyadenylation of Toll was competed with the PR, but not with the distal 119-nt fragment of the Toll 3' UTR that contained the CPE and the hexanucleotide (Fig. 4A, lanes 1-6). Conversely, the PR did not compete polyadenylation 
Coll et al.

A

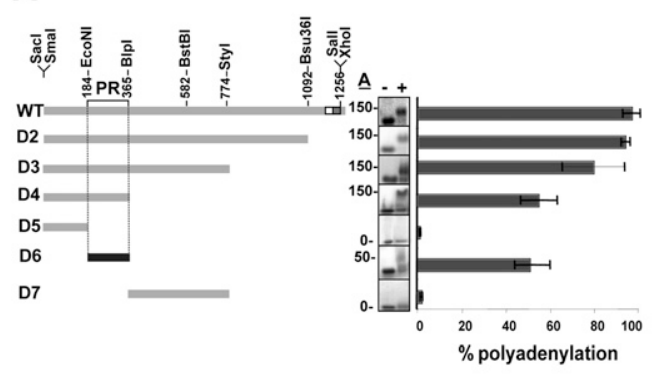

B

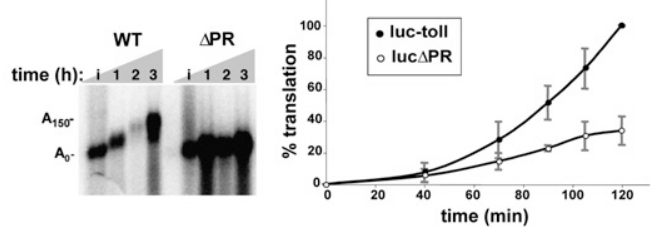

Figure 3. Elements required for cytoplasmic polyadenylation of Toll mRNA. (A, left panel) Schematic representation of Toll 3' UTR deletion derivatives. Nucleotide positions are shown according to the annotated Drosophila Toll sequence, taking as reference the first nucleotide of the 3' UTR. The location of unique restriction sites and the PR are indicated. Restriction sites at both ends of Toll belong to the vector in which this sequence was cloned. Typical patterns of these RNAs are shown in the middle panel, before $(-)$ or after $(+)$ incubation in the Drosophila extracts. The sizes of the respective poly(A) tails are indicated. (Right panel) Quantification of the efficiency of polyadenylation, measured as the percentage of RNA that was polyadenylated versus the total signal (polyadenylated and nonadenylated) within the " + " lane. Values represent the average of at least three independent experiments. (B) The PR is essential for polyadenylation and translation of Toll. (Left panel) Polyadenylation time course of a wild-type Toll 3' UTR or a mutant derivative lacking the PR. (Right panel) Translation efficiencies of reporter mRNAs containing either wild-type or $\triangle$ PR Toll 3' UTRs. Renilla luciferase mRNA was cotranslated as an internal control. The firefly values were corrected for Renilla expression, and the data are represented relative to the translation of Luc-toll mRNA at the last time point. Curves represent the average of three independent experiments using a single batch of extract.

of CAT-CycB1 (Fig. 4A, lanes 7-10), while polyadenylation of this transcript was efficiently competed by an excess of CycB1, as well as by any fragment of Toll containing the CPE and the hexanucleotide, including the full-length Toll 3' UTR (Fig. 4A, lanes 11-18). Consistent with the results of the polyadenylation assays, the PR competed translation of a Toll reporter but not of a CycB1 reporter (Supplemental Fig. 2). The PR competed both polyadenylation and translation less efficiently than the full-length Toll 3' UTR, in agreement with its lower polyadenylation efficiency. The competition results cannot be explained by different affinities of the same factors for the PR compared with the canonical sequences, because neither the PR competes CycB1 mRNA polyadenylation nor $C y c B 1$ competes Toll polyadenylation. Thus, we conclude that polyadenylation of Toll is driven by a complex that binds to the PR and differs from the canonical machinery in at least one limiting component.

Previously, a region of the Toll 3' UTR that lies downstream from the PR (located between nucleotides 582 and 774) was shown to promote polyadenylation of this transcript (Schisa and Strickland 1998). In our hands, this region could not compete for polyadenylation of Toll (data not shown), suggesting that it does not function for polyadenylation in early embryos. Nevertheless, it should be noted that translation of Toll is also required at later times in development, where these sequences and/or the canonical signals could become relevant.

To map more finely the sequences within the PR that were responsible for polyadenylation, we first searched for regions ultraconserved among Drosophilids. In addition, we looked for sequence words within the PR significantly overrepresented in the 3' UTRs of Drosophila melanogaster transcripts. We found two ultraconserved regions and two related sequence words distributed along the PR (Supplemental Fig. 3A,B, observe shadowed regions and words within red boxes). Fragments of the PR containing or lacking these sequences were used in functional competition assays (Supplemental Fig. $3 \mathrm{C}$ ). The results suggest the existence of a complex element responsible for polyadenylation of Toll that is not associated with a simple linear sequence. We speculate that a structure-or multiple redundant, interdependent linear sequences-within the PR are necessary for polyadenylation. Interestingly, the conserved region at the $3^{\prime}$ end of the PR consisting of TGTTATCTGTAAGC behaved as a stabilization element. All fragments containing this region destabilized Toll when added in excess, while fragments lacking it did not (Supplemental Fig. 3C).

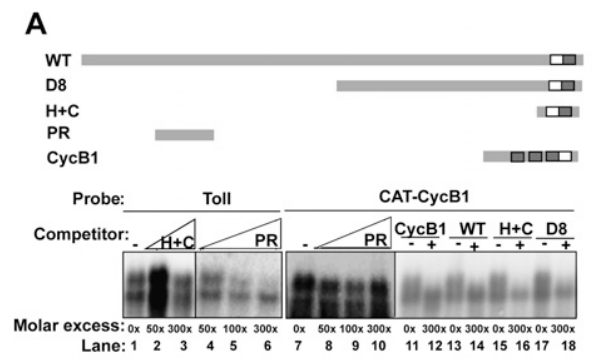

B
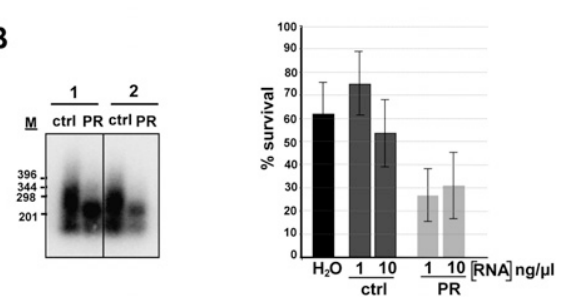

Figure 4. The PR is required for noncanonical cytoplasmic polyadenylation in vitro and in vivo. $(A)$ Polyadenylation competition experiments using the RNAs schematically represented in the top panel as competitors. Experiments were performed as indicated in the legend for Figure 2B. (B) Excess PRs disrupt polyadenylation of endogenous Toll and reduce viability. (Left panel) Embryos (0-30 min) were microinjected with different amounts of the PR or an unrelated RNA of similar length (159 nt) at a concentration of $10 \mathrm{ng} / \mu \mathrm{L}$. Samples were collected $1 \mathrm{~h}$ after injection, the RNA was extracted, and the poly(A) tail length was tested by PAT assay. Amplified products were visualized by Southern blot using a random-primed probe against the Toll 3' UTR. The results for two independent injections are shown. (Right panel) Embryos were microinjected with RNA solutions at different concentrations or with water as control, and survival was scored as the percentage of hatched embryos as indicated in the Materials and Methods. The average of at least three independent injections of 100 embryos per injection is shown. 
Importantly, fragment 9 (F9) strongly destabilized Toll but did not compete for polyadenylation, showing that polyadenylation and stability of Toll are separable processes.

We next wanted to test the relevance of the PR in vivo. We performed in vivo competition experiments by injecting wild-type early embryos with either the PR or an unrelated RNA of the same length as control. We then tested polyadenylation of endogenous Toll mRNA and survival of injected embryos, as timely expression of Toll is essential for early development. The results showed that the PR specifically competed polyadenylation of endogenous Toll and reduced the viability of early embryos (Fig. 4B). These results indicate that PR directs noncanonical polyadenylation in vitro and in vivo.

The Toll polyadenylation mechanism described here may affect a variety of mRNAs. Recent in silico EST database analysis indicates an incidence of the hexanucleotide $\mathrm{A} / \mathrm{A} / \mathrm{U}) \mathrm{UAAA}$ of $60 \%-70 \%$, suggesting that a significant fraction of mRNAs lack consensus hexanucleotide signals and may undergo AAUAAA-independent polyadenylation (for review, see MacDonald and Redondo 2002). In addition, activation of Drosophila pgc (polar granule component) mRNA in early embryos appears independent of Orb (Rangan et al. 2009).

Which factors could be involved in polyadenylation of Toll? Mutations in cortex and grauzone were identified genetically to affect polyadenylation of Toll (Lieberfarb et al. 1996). Cortex is an activator of the anaphasepromoting complex, and mutations in this gene prevent the completion of meiosis (Chu et al. 2001). On the other hand, Grauzone is a transcription factor necessary for activation of Cortex (Harms et al. 2000). Thus, Cortex and Grauzone may affect polyadenylation of Toll indirectly by precluding the normal initiation of embryogenesis. Similarly, embryos from wisp mutant mothers are defective in polyadenylation of several maternal mRNAs, including Toll (Cui et al. 2008). Wisp is present until $\sim 2 \mathrm{~h}$ of development and, therefore, could be directly involved in polyadenylation of Toll. However, Wisp is also required for expression of Cortex (Benoit et al. 2008), so it is unclear to what extent the observed effects on Toll polyadenylation are due to primary defects in Cortex expression. Direct biochemical dissection using the cell-free polyadenylation system, combined with Drosophila genetics, will allow us to decipher the components of both the CPE-dependent and CPE-independent cytoplasmic polyadenylation machineries.

\section{Materials and methods}

\section{Extract preparation}

Extracts were prepared from staged 90-min embryos as described in Gebauer et al. (1999). To stage embryos, collecting trays were exchanged every $90 \mathrm{~min}$, three times, and the third batch of trays was used to prepare extracts. After preparation, extracts were adjusted to $10 \%$ glycerol, snapfrozen in liquid nitrogen, and stored at $-80^{\circ} \mathrm{C}$.

\section{In vitro polyadenylation and translation}

Reactions using 90-min embryo extracts were assembled as described in Gebauer et al. (1999), without tRNA. In these reactions, both polyadenylation and translation could be observed. Typically, 0.01 pmol of substrate mRNA was used in the reaction. The use of small amounts of substrate is relevant, as we found that polyadenylation is saturable in this system. To account for batch-to-batch differences in the polyadenylation kinetics and efficiency, polyadenylation of each RNA construct was tested in different batches of extract, carrying in parallel the full-length Toll 3' UTR as a positive control. In some cases, Renilla mRNA was cotranslated as an internal control. After incubation, the translation efficiency was determined by measuring the luciferase activity using the Dual Luciferase Assay System (Promega), and firefly luciferase values were corrected for Renilla expression.

Polyadenylation was tested by either PAT assay, Northern blot, or direct visualization using radioactively labeled RNAs. For Northern blots, a random-primed probe against the full-length $3^{\prime}$ UTR of Toll was used. Radioactively labeled RNAs were resolved in denaturing $6 \%$ acrylamide gels and visualized in a PhosphorImager. PAT assays were performed as described by Sallés and Strickland (1995) after treatment of RNA samples with Turbo DNase (Ambion). The gene-specific oligonucleotides used for these assays are described in the Supplemental Material. To amplify endogenous Toll, RNA was extracted from embryos using Trizol (Invitrogen), and 150-300 ng of total RNA (100 embryos) were used in the reaction. Amplified products were resolved in 1\% agarose gels.

For competition assays, extracts were preincubated for $10 \mathrm{~min}$ on ice with increasing amounts of ApppG-capped RNA competitor. The remaining reagents needed for translation were subsequently added, and the reaction was further incubated for $10 \mathrm{~min}$ before adding the radioactively labeled substrate mRNA.

\section{Plasmids and in vitro transcription}

DNA constructs are detailed in the Supplemental Material. mRNAs were synthesized as described previously (Gebauer et al. 1999). mRNAs lacked a poly(A) tail and contained a ${ }^{7 \mathrm{~m}} \mathrm{GpppG}$ cap. RNAs used as competitors contained an ApppG cap. Cordycepin was incorporated to the $3^{\prime}$ end of Luc-toll mRNA with yeast poly(A) polymerase (GE Healthcare), following the recommendations of the vendor.

\section{Microinjections}

Oregon embryos (0-30 min old) were injected in a ventral-posterior position as described previously (Schisa and Strickland 1998). Embryos were allowed to develop for $72 \mathrm{~h}$ at $18^{\circ} \mathrm{C}$, and the number of hatched embryos was scored to estimate the percentage of viability. For PAT assays, microinjected embryos were allowed to develop for $1 \mathrm{~h}$ before extraction of RNA with Trizol (Invitrogen).

\section{Acknowledgments}

We thank Raul Méndez, Juan Valcárcel, Martine Simonelig, Josep Vilardell, and Antoine Graindorge for critically reading this manuscript and for useful suggestions. We also thank Cornelia de Moor and Raúl Méndez for CycB1 plasmids. This work was supported by grants BMC2003-04108 and BFU2006-01874 from the Spanish Ministry of Education and Science, and grant 2005SGR00669 from the Department of Universities, Information, and Sciences of the Generalitat of Catalunya (DURSI). F.G. is supported by the I3 Program of the Spanish Ministry of Education and Science.

\section{References}

Barnard DC, Ryan K, Manley JL, Richter JD. 2004. Symplekin and xGLD-2 are required for CPEB-mediated cytoplasmic polyadenylation. Cell 119: 641-651.

Belloc E, Pique M, Mendez R. 2008. Sequential waves of polyadenylation and deadenylation define a translation circuit that drives meiotic progression. Biochem Soc Trans 36: 665-670.

Benoit B, Mitou G, Chartier A, Temme C, Zaessinger S, Wahle E, Busseau I, Simonelig M. 2005. An essential cytoplasmic function for the nuclear poly(A) binding protein, $\mathrm{PABP} 2$, in poly(A) tail length control and early development in Drosophila. Dev Cell 9: 511-522.

Benoit P, Papin C, Kwak JE, Wickens M, Simonelig M. 2008. PAP- and GLD2-type poly(A) polymerases are required sequentially in cytoplasmic polyadenylation and oogenesis in Drosophila. Development 135: 19691979.

Castagnetti S, Ephrussi A. 2003. Orb and a long poly(A) tail are required for efficient oskar translation at the posterior pole of the Drosophila oocyte. Development 130: 835-843. 
Coll et al.

Chang JS, Tan L, Schedl P. 1999. The Drosophila CPEB homolog, orb, is required for oskar protein expression in oocytes. Dev Biol 215: 91-106.

Charlesworth A, Ridge JA, King LA, MacNicol MC, MacNicol AM. 2002. A novel regulatory element determines the timing of Mos mRNA translation during Xenopus oocyte maturation. EMBO I 21: 27982806.

Charlesworth A, Cox LL, MacNicol AM. 2004. Cytoplasmic polyadenylation element (CPE)- and CPE-binding protein (CPEB)-independent mechanisms regulate early class maternal mRNA translational activation in Xenopus oocytes. J Biol Chem 279: 17650-17659.

Charlesworth A, Wilczynska A, Thampi P, Cox LL, MacNicol AM. 2006. Musashi regulates the temporal order of mRNA translation during Xenopus oocyte maturation. EMBO J 25: 2792-2801.

Chen F, Wilusz J. 1998. Auxiliary downstream elements are required for efficient polyadenylation of mammalian pre-mRNAs. Nucleic Acids Res 26: 2891-2898.

Chu T, Henrion G, Haegeli V, Strickland S. 2001. Cortex, a Drosophila gene required to complete oocyte meiosis, is a member of the Cdc20/fizzy protein family. Genesis 29: 141-152.

Cui J, Sackton KL, Horner VL, Kumar KE, Wolfner MF. 2008. Wispy, the Drosophila homolog of GLD-2, is required during oogenesis and egg activation. Genetics 178: 2017-2029.

Gebauer F, Corona DF, Preiss T, Becker PB, Hentze MW. 1999. Translational control of dosage compensation in Drosophila by Sex-lethal: Cooperative silencing via the $5^{\prime}$ and $3^{\prime}$ UTRs of msl-2 mRNA is independent of the poly(A) tail. EMBO J 18: 6146-6154.

Groisman I, Jung MY, Sarkissian M, Cao Q, Richter JD. 2002. Translational control of the embryonic cell cycle. Cell 109: 473-483.

Harms E, Chu T, Henrion G, Strickland S. 2000. The only function of Grauzone required for Drosophila oocyte meiosis is transcriptional activation of the cortex gene. Genetics 155: 1831-1839.

Juge F, Zaessinger S, Temme C, Wahle E, Simonelig M. 2002. Control of poly(A) polymerase level is essential to cytoplasmic polyadenylation and early development in Drosophila. EMBO I 21: 6603-6613.

Keleman K, Kruttner S, Alenius M, Dickson BJ. 2007. Function of the Drosophila CPEB protein Orb2 in long-term courtship memory. Nat Neurosci 10: 1587-1593.

Kim JH, Richter JD. 2006. Opposing polymerase-deadenylase activities regulate cytoplasmic polyadenylation. Mol Cell 24: 173-183.

Lieberfarb ME, Chu T, Wreden C, Theurkauf W, Gergen JP, Strickland S. 1996. Mutations that perturb poly(A)-dependent maternal mRNA activation block the initiation of development. Development 122: $579-588$.

MacDonald CC, Redondo JL. 2002. Reexamining the polyadenylation signal: Were we wrong about AAUAAA? Mol Cell Endocrinol 190: 1-8.

McGrew LL, Richter JD. 1990. Translational control by cytoplasmic polyadenylation during Xenopus oocyte maturation: Characterization of cis and trans elements and regulation by cyclin/MPF. EMBO $I \mathbf{9 :}$ 3743-3751.

Minshall N, Reiter MH, Weil D, Standart N. 2007. CPEB interacts with an ovary-specific eIF4E and 4E-T in early Xenopus oocytes. $J$ Biol Chem 282: 37389-37401.

Paillard L, Maniey D, Lachaume P, Legagneux V, Osborne HB. 2000. Identification of a C-rich element as a novel cytoplasmic polyadenylation element in Xenopus embryos. Mech Dev 93: 117-125.

Piqué M, Lopez JM, Foissac S, Guigo R, Mendez R. 2008. A combinatorial code for CPE-mediated translational control. Cell 132: 434-448.

Radford HE, Meijer HA, de Moor CH. 2008. Translational control by cytoplasmic polyadenylation in Xenopus oocytes. Biochim Biophys Acta 1779: 217-229.

Ralle T, Gremmels D, Stick R. 1999. Translational control of nuclear lamin B1 mRNA during oogenesis and early development of Xenopus. Mech Dev 84: 89-101.

Rangan P, DeGennaro M, Jaime-Bustamante K, Coux RX, Martinho RG, Lehmann R. 2009. Temporal and spatial control of germ-plasm RNAs. Curr Biol 19: 72-77.

Richter JD. 2007. CPEB: A life in translation. Trends Biochem Sci 32: 279-285.

Sallés FJ, Strickland S. 1995. Rapid and sensitive analysis of mRNA polyadenylation states by PCR. PCR Methods Appl 4: 317-321.

Sallés FJ, Lieberfarb ME, Wreden C, Gergen JP, Strickland S. 1994. Coordinate initiation of Drosophila development by regulated polyadenylation of maternal messenger RNAs. Science 266: 1996-1999.
Schisa JA, Strickland S. 1998. Cytoplasmic polyadenylation of Toll mRNA is required for dorsal-ventral patterning in Drosophila embryogenesis. Development 125: 2995-3003.

Simon R, Tassan JP, Richter JD. 1992. Translational control by poly(A) elongation during Xenopus development: Differential repression and enhancement by a novel cytoplasmic polyadenylation element. Genes \& Dev 6: 2580-2591.

Stebbins-Boaz B, Cao Q, de Moor CH, Mendez R, Richter JD. 1999. Maskin is a CPEB-associated factor that transiently interacts with elF-4E. Mol Cell 4: 1017-1027.

Vardy L, Orr-Weaver TL. 2007. The Drosophila PNG kinase complex regulates the translation of cyclin B. Dev Cell 12: 157-166.

Venkataraman K, Brown KM, Gilmartin GM. 2005. Analysis of a noncanonical poly(A) site reveals a tripartite mechanism for vertebrate poly(A) site recognition. Genes \& Dev 19: 1315-1327.

Wang YY, Charlesworth A, Byrd SM, Gregerson R, MacNicol MC, MacNicol AM. 2008. A novel mRNA 3' untranslated region translational control sequence regulates Xenopus Weel mRNA translation. Dev Biol 317: 454-466.

Zarudnaya MI, Kolomiets IM, Potyahaylo AL, Hovorun DM. 2003. Downstream elements of mammalian pre-mRNA polyadenylation signals: Primary, secondary and higher-order structures. Nucleic Acids Res 31: 1375-1386. 


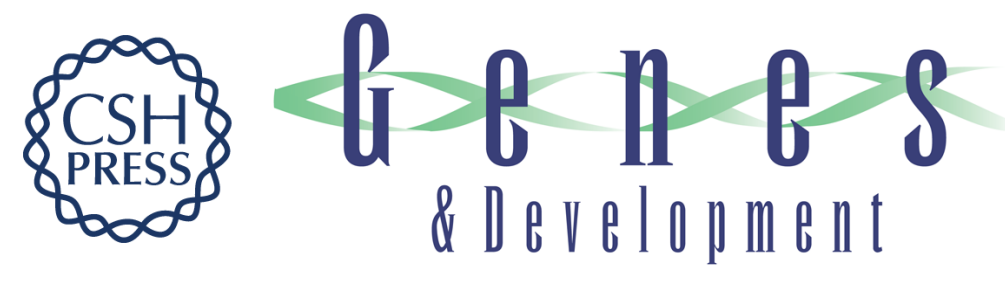

\section{A novel, noncanonical mechanism of cytoplasmic polyadenylation operates in Drosophila embryogenesis}

Olga Coll, Ana Villalba, Giovanni Bussotti, et al.

Genes Dev. 2010, 24:

Access the most recent version at doi:10.1101/gad.568610

Supplemental http://genesdev.cshlp.org/content/suppl/2009/12/29/24.2.129.DC1
Material

References This article cites 37 articles, 10 of which can be accessed free at:

http://genesdev.cshlp.org/content/24/2/129.full.html\#ref-list-1

License

Email Alerting Receive free email alerts when new articles cite this article - sign up in the box at the top

Service

right corner of the article or click here.

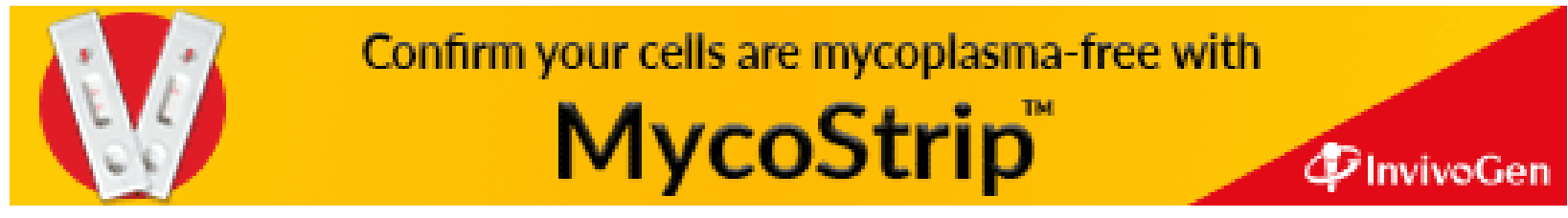

